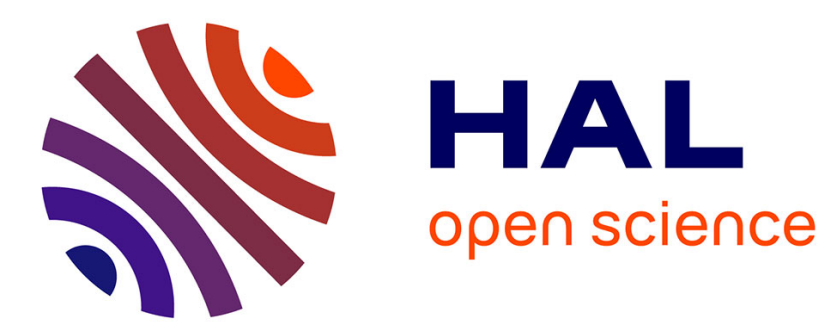

\title{
Kalman filtering for carrier phase recovery in optical offset-QAM Nyquist WDM systems
}

\author{
Trung Hien Nguyen, Christophe Peucheret
}

\section{To cite this version:}

Trung Hien Nguyen, Christophe Peucheret. Kalman filtering for carrier phase recovery in optical offset-QAM Nyquist WDM systems. IEEE Photonics Technology Letters, 2017, 29 (12), pp.10191022. 10.1109/LPT.2017.2701907 . hal-01533974

\section{HAL Id: hal-01533974 \\ https://hal.science/hal-01533974}

Submitted on 6 Jun 2017

HAL is a multi-disciplinary open access archive for the deposit and dissemination of scientific research documents, whether they are published or not. The documents may come from teaching and research institutions in France or abroad, or from public or private research centers.
L'archive ouverte pluridisciplinaire HAL, est destinée au dépôt et à la diffusion de documents scientifiques de niveau recherche, publiés ou non, émanant des établissements d'enseignement et de recherche français ou étrangers, des laboratoires publics ou privés. 


\title{
Kalman Filtering for Carrier Phase Recovery in Optical Offset-QAM Nyquist WDM Systems
}

\author{
Trung-Hien Nguyen, Christophe Peucheret
}

\begin{abstract}
Nyquist wavelength-division-multiplexing (NWDM) systems based on offset quadrature amplitude modulation (OQAM) have been considered as interesting candidates to achieve a better spectral efficiency. However, such systems are very sensitive to laser phase noise that causes the loss of OQAM orthogonality. In this paper, we investigate carrier phase recovery using a modified extended Kalman filter (M-EKF) in OQAM-based NWDM systems. The proposed method can achieve an optical signal-to-noise ratio (OSNR) penalty lower than that of the state-of-the-art modified blind phase search (MBPS) algorithm. More specifically, for a $10^{-5}$ normalized laser linewidth, the M-EKF method exhibits 0.8-dB and 1.7-dB OSNR penalties (at a bit-error-rate of $3.8 \times 10^{-3}$ ) lower than the M-BPS method for 16- and 64-OQAM modulations, respectively. Furthermore, compared to the M-BPS method, our proposed method is of lower complexity. The required total number of multiplications and additions of the M-EKF method is reduced by about 3.3 and 6.7 times compared to that of M-BPS for 16and 64-OQAM modulations, respectively, confirming the effectiveness of the proposed method.
\end{abstract}

Index Terms - Nyquist WDM, offset-QAM, coherent detection, carrier phase recovery, Kalman filtering.

\section{INTRODUCTION}

$\mathrm{I}^{2}$ $\mathrm{N}$ ORDER TO meet the growing traffic demand, increasing the spectral efficiency (SE) of optical fiber communications is a key factor. In Nyquist wavelength-division-multiplexing (NWDM) systems, offset quadrature amplitude modulation (OQAM) has recently been used to allow for a channel spacing equal to the symbol rate, thus providing an optimized SE [1-3]. Compared to QAM-based NWDM systems, OQAMbased NWDM systems enable the spectra of adjacent channels to overlap without any crosstalk. This is made possible thanks to a half-symbol time delay between the in-phase (I) and quadrature (Q) components of OQAM signals. However, under the impact of laser phase noise (PN), OQAM signals suffer not only from constellation rotation but also from additional crosstalk [3]. Therefore, the laser linewidth requirement is very stringent in OQAM-based NWDM systems. For example, earlier work [1-2] used a laser linewidth of only a few $\mathrm{kHz}$ for the validation of OQAMbased NWDM systems.

Trung-Hien Nguyen is with OPERA Department, Universite libre de Bruxelles, 1050 Brussels, Belgium (e-mail: trung-hien.nguyen@ulb.ac.be).

Christophe Peucheret is with FOTON Laboratory, CNRS, University of Rennes 1, F-22305 Lannion, France.
Carrier phase recovery (CPR) is an indispensable block of receiver-side digital signal processing (DSP). Some CPR methods are now well-established for QAM constellations. However, they should be revisited in order to compensate for the effect of PN in OQAM modulation. Some CPR solutions have already been proposed for OQAM modulation [3-6]. Among them, modified blind phase search (M-BPS) has been considered as the most efficient CPR algorithm [3]. However, the computation effort (CE) increases linearly with the OQAM modulation order, even though some simplified versions have recently been proposed [4-5]. CPR based on constellation classification for OQAM signals has recently been proposed in [6]. Although this method offers reduced CE, it operates in the feedback manner and suffers from classification errors when dealing with higher modulation orders such as 64-OQAM. Note that, the principle of M-BPS is based on only phase rotation tests in order to estimate the PN value, whereas the received symbols are actually corrupted by both amplitude and phase noise, making M-BPS less effective, especially with high OQAM orders.

Extended Kalman filtering (EKF) provides optimal phase tracking and amplitude noise suppression in the minimum mean squared error sense and its effectiveness has recently been studied in coherent QAM systems [7]. In this paper, a modified EKF (M-EKF) is proposed for CPR in OQAM-based NWDM systems. The proposed method is numerically validated in a 5-channel NWDM system based on 16- and 64-OQAM signals. Even though state-of-the-art CPR methods claim to be suitable for any $M$-OQAM modulation, their validation has been limited, to the best of our knowledge, to only 16-OQAM modulation. CPR is evaluated in this paper for up to 64-OQAM signals in an NWDM system for the first time, and compared to the state-of-the-art M-BPS method. The results show that M-EKF exhibits an optical signal-to-noise ratio (OSNR) penalty lower than that of M-BPS at a bit-errorrate (BER) of $3.8 \times 10^{-3}$ (corresponding to the soft forwarderror-correction (FEC) limit [8]), while keeping a low complexity.

\section{NYQUiST WDM SYSTEM BASED ON OFFSET QAM}

In order to focus on the performance assessment of the proposed $\mathrm{M}-\mathrm{EKF}$, only one polarization is considered and other impairments (i.e. chromatic dispersion, frequency offset) are assumed to be completely compensated elsewhere. The system model is based on the NWDM system described in $[3,5,6]$, as shown in Fig. 1(a). The multi-carrier source has a 

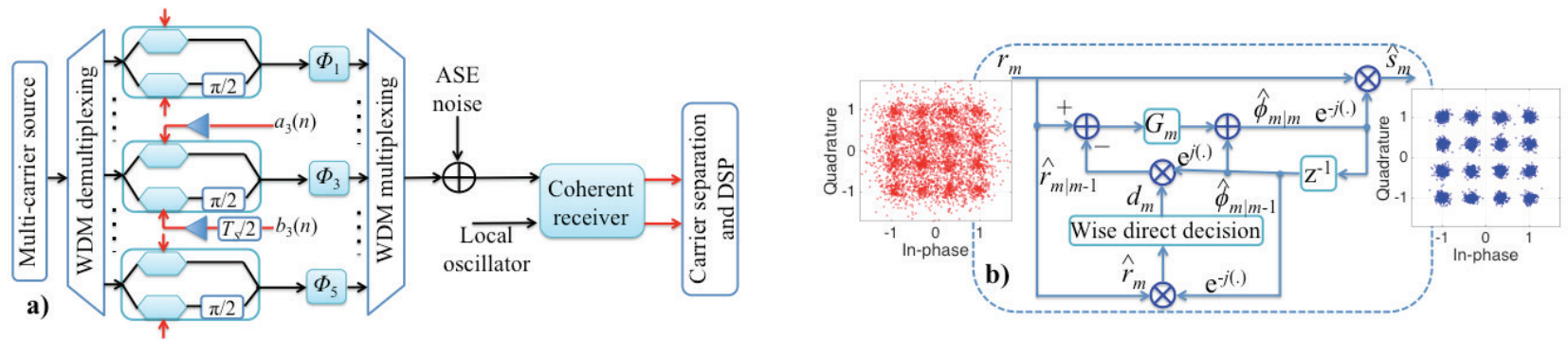

Fig. 1. a) NWDM-OQAM system model, considering one polarization and perfect channel impairments compensation. ASE: amplified spontaneous emission. DSP: digital signal processing. b) Block diagram of modified extended Kalman filter (M-EKF) for OQAM signals. Insets: constellations of 16-OQAM (at the symbol rate) with normalized laser linewidth $\Delta v \cdot T_{S}=2 \times 10^{-5}$ without (left) and with (right) M-EKF compensation for OSNR $=17.8 \mathrm{~dB}$.

frequency spacing of $30 \mathrm{GHz}$, which is equal to the symbol rate per optical carrier. Five optical carriers are utilized in our simulations as the inputs of the IQ modulators. A pseudorandom binary sequence (PRBS) with a length of $2^{17}-1$ is used and mapped on the QAM constellation and a root-raisedcosine (RRC) filter with a roll-off factor of 0.4 is applied to shape the signals. The QAM symbols are differentially encoded [9] in order to suppress the phase ambiguity inherent to the M-BPS algorithm. The I and Q components are then temporally offset by $T_{S} / 2$, where $T_{S}$ is the symbol duration, before being applied to the IQ modulator. Before wavelengthmultiplexing, the modulated optical signals are phase shifted by $\Phi_{k}=(k-3) \times \pi / 2$, where $k=1, \ldots, 5$, to ensure optimal performance of the NWDM system [3]. The signal is then corrupted by amplified spontaneous emission (ASE) noise modeled as zero-mean additive white Gaussian noise (AWGN). At the receiver side, the local oscillator laser acts as a source of PN that needs to be compensated by the CPR. The signal is then coherently detected and sampled with two samples per symbol. The even sample $R_{l, m}^{0}$ and odd sample $R_{l, m}^{1}$ of the $l$-th channel can be represented as [3]

$$
\begin{aligned}
& R_{l, m}^{0} \propto\left(a_{l, m} I_{l, l}(0)+j b_{l, m} Q_{l, l}(0)\right) \exp \left(j \varphi_{l}\right)+w_{l, m}^{0} \\
& R_{l, m}^{1} \propto\left(a_{l, m} I_{l, l}\left(0.5 T_{S}\right)+j b_{l, m} Q_{l, l}\left(0.5 T_{S}\right)\right) \exp \left(j \varphi_{l}\right)+w_{l, m}^{1}
\end{aligned}
$$

where $a_{l, m}$ and $b_{l, m}$ are the $m$-th logic data of the respective I and Q components of the $l$-th channel, $I_{k, l}$ and $Q_{k, l}$ denote the impulse responses of the I and Q tributaries of the $k$-th channel after wavelength division demultiplexing, respectively, and $\varphi_{l}$ is the phase of the $l$-th channel. The intrinsic interference originating from the neighboring symbols on the channel of interest is represented by $w_{l, m}^{p}$, where $p=0,1$. Finally, the recovered sample $r_{l, m}$ can be deduced by [3]

$$
r_{l, m}=\operatorname{Re}\left(R_{l, m}^{0}\right)+j \operatorname{Im}\left(R_{l, m}^{1}\right),
$$

in which $\operatorname{Re}$ and $\mathrm{Im}$ denote the real and imaginary part operators, respectively. As pointed out in $[3,6]$, the transmitted signal can be recovered without any crosstalk in the absence of PN. For the sake of simplicity, the channel index is omitted in the next section. The impact of analog-to-digital converters and digital-to-analog converters on higher-frequency channels is ignored in what follows. Since the phase shifts between channels are instrumental in suppressing ICI in NWDM systems, it is essential to identify the correct starting position of the transmitted signals at the receiver side in order to shift back the phase difference in the DSP stage. We assume that the use of a training sequence, i.e. Zadoff-Chu sequence [6], can correctly identify the starting position of the signal. Moreover, it can be used to estimate the initial PN value for the following CPR. Without loss of generality, we assume that the initial PN value is set to zero in the simulations.

\section{CARrier Phase Recovery BASEd on EXTENDED KALMAN FILTER}

The PN is modeled as a discrete random time walk $\varphi_{m}=\varphi_{m-1}+\Theta_{m}$, where $\Theta_{m}$ denotes the PN variation on the sample $m$, which is modeled as a zero-mean Gaussian random variable of variance $2 \pi \cdot \Delta v \cdot T_{S}$. The parameter $\Delta v$ denotes the laser linewidth. In order to apply the EKF to compensate for PN in NWDM systems, we first build the process and measurement equations. The $m$-th received sample at twice the symbol rate, $r_{m}$, can be rewritten as

$$
r_{m}=c_{m} \exp \left(j \varphi_{m}\right)+w_{m},
$$

where $c_{m}$ is the real filtered transmitted symbol from $M$-ary pulse amplitude modulation ( $M$-PAM) constellation, inherent to the OQAM staggering process. $w_{m}$ describes the collective effect of amplifier noise and interferences from adjacent channels and is assumed to be zero-mean AWGN. In the high OSNR regime, the received samples can be represented by $r_{m}=c_{m} \exp \left(j \phi_{m}\right)$, where $\phi_{m}=\left[\varphi_{m}+\operatorname{Im}\left(w_{m}\right)\right]+j \operatorname{Re}\left(w_{m}\right)$ [7]. By estimating the complex quantity $\phi_{m}$, both the amplitude and phase noises can be mitigated. Inspired from [7], we here propose to use an M-EKF to estimate $\phi_{m}$ for OQAM signals. As discussed in [7], two main equations in M-EKF are given by

$$
\begin{aligned}
& \phi_{m}=\phi_{m-1}+\omega_{m} \\
& r_{m}=c_{m} \exp \left(j \phi_{m}\right)+\gamma_{m},
\end{aligned}
$$

where $\omega_{m}\left(\gamma_{m}\right)$ is the process (measurement) noise whose correlation matrix is $Q(R)$. Fig. 1(b) presents the block diagram of the proposed M-EKF algorithm. The variables with the subscripts $m \mid m-1$ present a priori estimates (or predictions) to the availability of the current $m$-th observation and the ones with the subscripts $m \mid m$ (or $m$ ) denotes $a$ posteriori estimates (or updates) when the current $m$-th observation is available [7]. In principle, the optimal phase estimate $\hat{\phi}_{m \mid m}$ is calculated by combining the prediction $\hat{\phi}_{m \mid m-1}$ and the Kalman gain, $G_{m}$, so-called weighted innovation, which is the difference between the received sample and its rotated version of the wise direct decision, $d_{m}$. As opposed to the direct decision used in [7], the wise direct decision (WD) 
TABLE I: EXTENDED KALMAN FILTERING ALGORITHM

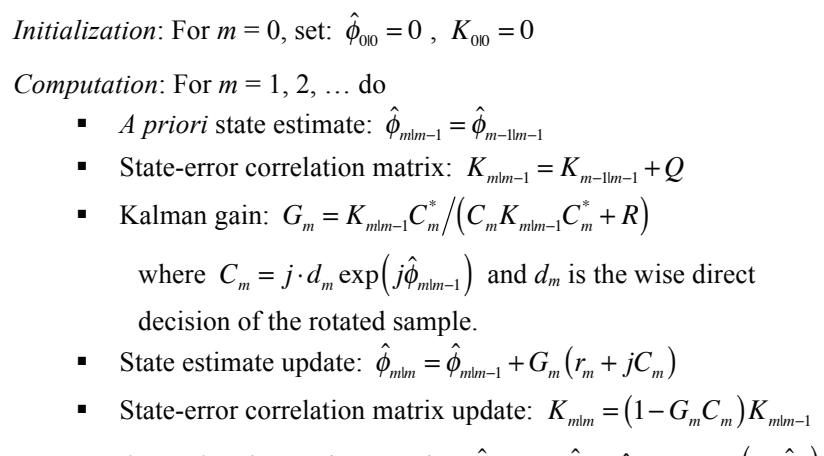

Recovered samples: de-rotating $r_{m}$ using $\hat{\phi}_{m \mid m}$ or $\hat{\phi}_{m}: \hat{s}_{m}=r_{m} \exp \left(-j \hat{\phi}_{m}\right)$

$Q(R)$ is the correlation matrix of the process (measurement) noise.

operation is as follows

$d_{m}=\left\{\begin{array}{c}W D\left(\operatorname{Re}\left(\hat{r}_{m}\right)\right)+j \operatorname{Im}\left(\hat{r}_{m}\right), \text { odd samples } \\ \operatorname{Re}\left(\hat{r}_{m}\right)+j W D\left(\operatorname{Im}\left(\hat{r}_{m}\right)\right), \text { even samples }\end{array}\right.$

where $\hat{r}_{m}=r_{m} \exp \left(-j \hat{\phi}_{m \mid m-1}\right)$ is the rotated version of $r_{m}$ by the predicted phase estimate. Note that, we de-stagger and downsample the PN-compensated samples to the symbol rate before the BER calculation and constellation presentation. Compared to the constellations illustrated in $[5,6]$, where the phase noise value is fixed, our constellations are presented with random PN (simulated as a Wiener process) that impacts differently each sample. The insets of Fig. 1(b) show examples of 16-OQAM constellations at the symbol rate with a normalized laser linewidth (NLW) $\Delta v T_{S}=2 \times 10^{-5}$ without and with applying M-EKF when the OSNR of the NWDM signals at the coherent receiver input is $17.8 \mathrm{~dB}$. It is clearly seen that, even though the constellation before applying the CPR is corrupted due to $\mathrm{PN}$-induced crosstalk, it can be recovered by the M-EKF, showing the effectiveness of the proposed method. The details of the M-EKF algorithm are summarized in Tab. I. Considering the two main equations of M-EKF, only the measurement equation is nonlinear. It needs to be linearized by taking its first derivative around the a priori state estimate $\hat{\phi}_{m \mid m-1}$, resulting in the linearized measurement matrix $C_{m}=j \cdot d_{m} \exp \left(j \hat{\phi}_{m \mid m-1}\right)$, which is used for the Kalman gain calculation and the state updates as shown in Tab. I. Furthermore, we only use the past phase estimate to obtain the current phase estimate, which further distinguishes our proposal from the one in [7]. It should be noted that the Kalman gain is a complex number enabling the effective compensation of either amplitude or phase noise [7]. As will be shown later, this key advantage will translate into M-EKF presenting a lower OSNR penalty compared to M-BPS, where only phase rotation is considered for PN compensation.

\section{RESUlTS AND Discussion}

The performance of the proposed CPR method is numerically studied with a 5-channel NWDM optical coherent system based on 16- and 64-OQAM modulations and compared to the performance of the M-BPS algorithm. Note
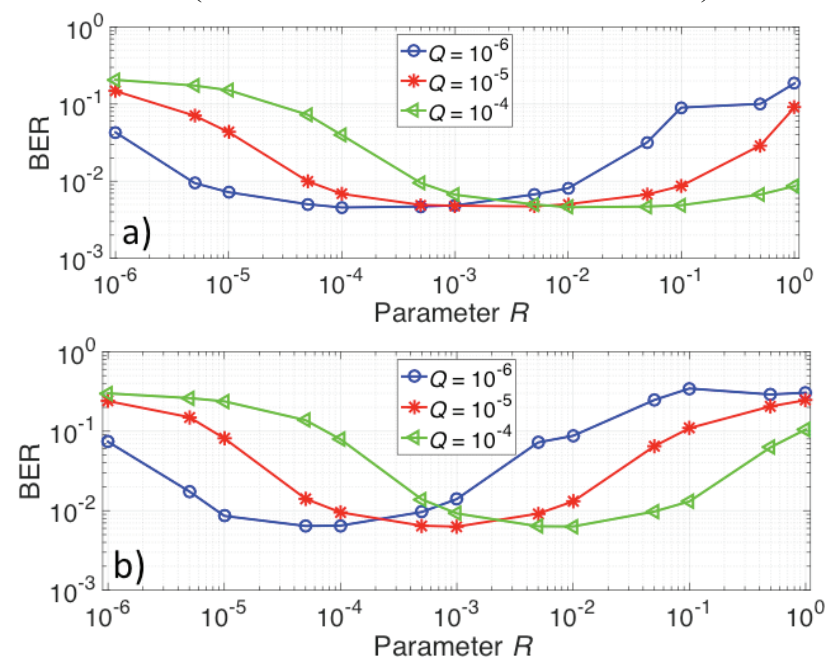

Fig. 2. BER as a function of the $R$ value at OSNRs of a) $17 \mathrm{~dB}$ for 16-OQAM and b) $23.3 \mathrm{~dB}$ for 64-OQAM modulations. $\Delta v \cdot T_{S}=2 \times 10^{-5}$.

that differential decoding [9] of the recovered symbols is carried out before BER calculation over about 130000 symbols. However, differential en(de)coding is only required for the M-BPS to solve the phase ambiguity problem. The M-EKF, updating the PN progressively, does not result in phase ambiguity problems. The coding step can be avoided in this case. Hence, the overall system complexity is reduced when using M-EKF instead of M-BPS for PN compensation. For a fair comparison, the number of phase tests of the M-BPS algorithm is set to 32 and 64 for 16- and 64-OQAM signals, respectively, whereas the block length is chosen to be 20 for both modulation formats, in order to achieve optimal performance [4-5].

In the M-EKF algorithm, the correlation matrix of the process (measurement) noise $Q(R)$ specifies the Kalman gain and depends on the noise level [7]. In practice, the noise level is not always available, even though it can be estimated by various techniques. For this reason, we first optimize $Q(R)$ at OSNR values of $17 \mathrm{~dB}$ and $23.3 \mathrm{~dB}$ (OSNR values corresponding to the soft FEC limit) for 16- and 64-OQAM signals, respectively. Note that, the OSNR is defined as the ratio between the average symbol power at the coherent receiver input and the noise power in $0.1-\mathrm{nm}$ bandwidth at 1550 -nm wavelength. The $Q(R)$ values that minimize the BER are selected. Fig. 2(a) and (b) present the BER as a function of the $R$ value for 16- and 64-OQAM signals, respectively, when $Q$ is set to different values and $\Delta v T_{S}=2 \times 10^{-5}$. When $Q$ is varied, the value of $R$ that minimizes the BER scales by the same amount as the $\mathrm{Q}$ value itself. In the following simulations, we choose $(Q, R)$ sets of $\left(10^{-6}, 10^{-4}\right)$ and $\left(10^{-6}\right.$, $5 \times 10^{-4}$ ) for 16 - and 64-OQAM signals, respectively. Note

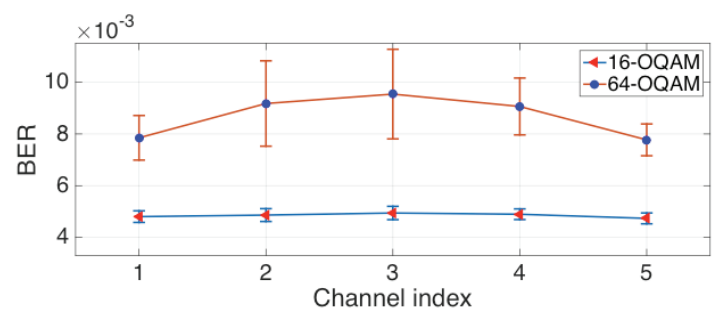

Fig. 3. BERs of the different channels at OSNR values of $17 \mathrm{~dB}$ and $23.3 \mathrm{~dB}$ for 16- and 64-OQAM, respectively, and their standard deviation calculated over 50 simulation runs. 
$>$ REPLACE THIS LINE WITH YOUR PAPER IDENTIFICATION NUMBER (DOUBLE-CLICK HERE TO EDIT) $<$
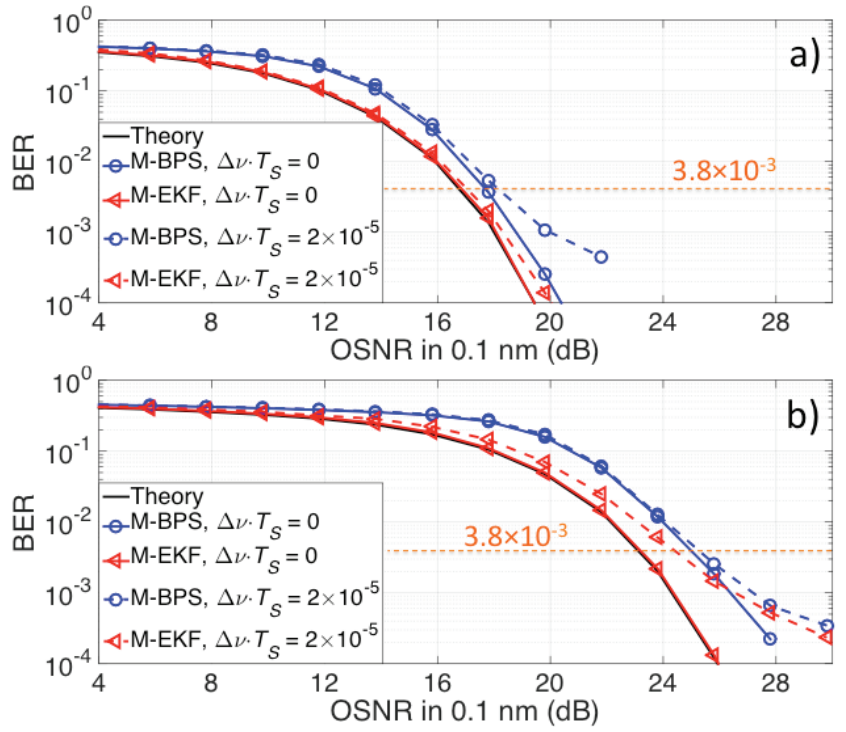

Fig. 4. BER versus OSNR for a) 16-OQAM and b) 64-OQAM modulations.

that, the optimal $Q(R)$ values change only slightly for different OSNRs. Fig. 3 presents the average BERs of the different channels and their standard deviations calculated over 50 simulation runs. The calculated BER varies only slightly over the channels. Consequently we describe the system performance by its average BER over the 5 channels.

The evolutions of the BER at different OSNRs are presented in Fig. 4(a) and (b) for 16- and 64-OQAM signals, respectively and compared to reference curves (labelled "theory") corresponding to an ideal (no phase noise) AWGN channel [9]. First, BER curves were computed using the two algorithms when $\Delta v T_{S}=0$ and $\Delta v T_{S}=2 \times 10^{-5}$. In the absence of PN, M-EKF shows no OSNR penalty, whereas M-BPS exhibits some OSNR penalties compared to the theory curve. The OSNR penalties increase when considering a higher modulation order. Those penalties are due to the discretization error in the M-BPS algorithm. In the presence of PN, M-EKF outperforms M-BPS regardless of the OSNR for both modulations. More specifically, for 64-OQAM signals, M-EKF exhibits a $1.3 \mathrm{~dB}$ OSNR penalty at a BER of $3.8 \times 10^{-3}$, whereas the penalty presents a higher value of $2.3 \mathrm{~dB}$ for M-BPS. This is due to the fact that the higher modulation format order introduces more amplitude noise when using OQAM signals, making PN compensation based on only phase rotation used in M-BPS less effective compared to the simultaneous amplitude and PN compensation by MEKF.

OSNR penalties compared to the reference OSNR (AWGN only) at a BER of $3.8 \times 10^{-3}$, are further investigated in Fig. 5 as a function of the NLW. It can be seen that, when the NLW is smaller than $3 \times 10^{-5}$, the OSNR penalty of M-EKF is smaller than that of M-BPS. For instance, at a $10^{-5} \mathrm{NLW}$, the M-EKF method exhibits $0.8-\mathrm{dB}$ and $1.7-\mathrm{dB}$ lower OSNR penalties compared to the M-BPS for 16- and 64-OQAM modulation, respectively. The M-BPS method can tolerate a larger NLW than M-EKF at the expense of higher OSNR penalties.

The complexity of the algorithms is estimated by counting the number of real multiplications, real additions and decisions per symbol required to compensate for the PN. Assuming that

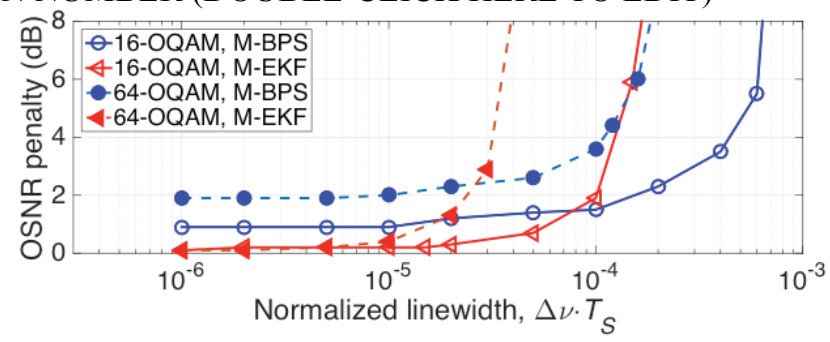

Fig. 5. OSNR penalty at a $3.8 \times 10^{-3} \mathrm{BER}$ versus the normalized linewidth.

complex multiplications require 4 real multiplications and 2 additions, the complexity comparison between M-EKF and M-BPS is summarized in Tab. II. Due to the fact that the number of phase tests used in M-BPS is at least equal to 32 in order to ensure optimum performance, the required total number of multiplications and additions of the M-EKF method is less than that of the M-BPS method (i.e. about 3.3 and 6.7 times reduction for 16- and 64-OQAM, respectively).

TABLE II: COMPLEXITY COMPARISON PER SYMBOL

\begin{tabular}{cccc}
\hline \hline Method & Real additions & Real multiplications & Decision \\
\hline M-BPS & $2 B$ & $3 B$ & $2 B$ \\
M-EKF & 20 & 28 & 1 \\
\hline \hline
\end{tabular}

\section{CONCLUSION}

A low complexity M-EKF algorithm for OQAM signals has been proposed and numerically validated in a 5-channel Nyquist WDM system. Compared to the M-BPS method, the proposed method can reduce the OSNR penalty at a $3.8 \times 10^{-3}$ BER thanks to its simultaneous mitigation of the amplitude and phase noises. Moreover, M-EKF exhibits a lower complexity compared to M-BPS, confirming the effectiveness of the proposed method.

\section{REFERENCES}

[1] J. Zhao, and A. D. Ellis, "Offset-QAM based coherent WDM for spectral efficiency enhancement," Opt. Epress, vol. 19, no. 15, pp. 14617-14631, Jul. 2011.

[2] S. Randel, A. Sierra, X. Liu, and P. J. Winzer, "Study of multicarrier offset-QAM for spectrally efficient coherent optical communications," in Proc. ECOC, 2011, p. Th.11.A.1.

[3] H. Tang, M. Xiang, S. Fu, M. Tang, P. Shum, and D. Liu, "Feed-forward carrier phase recovery for offset-QAM Nyquist WDM transmission," Opt. Express, vol. 23, no. 5, pp. 6215-6227, Mar. 2015.

[4] T.-H. Nguyen, S.-P. Gorza, J. Louveaux, and F. Horlin, "Lowcomplexity blind phase search for filter bank multicarrier offset-QAM optical fiber systems," in Proc. SPPcom 2016, Vancouver, Canada, Jul. 2016, p. SpW2G.2.

[5] J. Lu, et al., "Vertical blind phase search for low-complexity carrier phase recovery of offset-QAM Nyquist WDM transmission," Opt. Commun., vol. 382, pp. 212-218, Jan. 2017.

[6] H. Tang, S. Fu, H. Liu, M. Tang, P. Shum, and D. Liu, "Low-complexity carrier phase recovery based on constellation classification for M-ary offset-QAM signal," J. Lightw. Technol., vol. 34, no. 2, pp. 1133-1140, January 2016.

[7] L. Pakala, and B. Schmauss, "Extended Kalman filtering for joint mitigation of phase and amplitude noise in coherent QAM systems," Opt. Express, vol. 24, no. 6, pp. 6391-6401, Mar. 2016.

[8] A. Leven, F. Vacondio, L. Schmalen, S. T. Brink, and W. Idler, "Estimation of soft FEC performance in optical transmission experiments," IEEE Photon. Technol. Lett., vol. 23, no. 20, pp. 15471549, October 2011.

[9] J. K. Hwang, Y.-L. Chiu, and C.-S. Liao, "Angle differential-QAM for resolving phase ambiguity in continuous transmission system," Int. J. Commun. Syst., vol. 21, pp. 631-641, Dec. 2007. 